\title{
Sternal Wound Complications: Objective Reclassification and Surgical Reconsideration
}

\author{
Ahmed Abdelrahman Elassal, MD, ${ }^{1,2}$ Khalid Ebrahim Al-Ebrahim, FRCSC, ${ }^{1}$ \\ Osman Osama Al-Radi, FRCSC, ${ }^{1}$ Husain Hamza Jabbad, FRCSC ${ }^{1}$ Osama Saber Eldib, MD ${ }^{2}$
}

${ }^{1}$ King Abdulaziz University Hospital, Cardiac Surgery Unit, Jeddah, Saudi Arabia; ${ }^{2}$ Zagazig University Hospital, Cardiothoracic Surgery Department, Zagazig, Egypt

\section{ABSTRACT}

Background: Sternal wound complications pose a tremendous challenge post-cardiac surgery. There's no consensus or clear guidelines to deal with them. We propose that simple and more objective classification helps to organize the range of sternal wound complications and suggest a relevant treatment strategy.

Methods: One-hundred-sixteen cases of sternal wound complications retrospectively were reviewed out of 2,391 adult patients, who underwent full sternotomy during cardiac surgery from 2006 to 2018. Eighty-six cases conservatively were managed and the remaining 30 cases required surgical intervention. More objective classification was proposed and less invasive fasciocutaneous flap was considered for nearly all reconstructive procedures.

Results: The incidence of sternal wound complications was $4.8 \%$. Conservative management was adopted for 86 cases, mean duration was $11.19 \pm 9.8$ days. Surgical management was performed in 30 patients $(25.86 \%) ; 28(93.3 \%)$ of whom recovered with good outcomes with less invasive fasciocutaneous flap done for 13 cases. Two cases had recurrence; one conservatively was managed and other was reoperated and healed well. The most common organisms in recurrent infections were N. coagulase (29.8\%), Klebsiella (12.5\%), pseudomonas (10.5\%), and MRSA (10.5\%). We had 4 mortalities. None of the mortalities were related to sternal wound complications; one was related to the cardiac surgery.

Conclusions: Sternal wound complications are grave events. Objective classification and proper management selection will gain better outcomes.

\section{INTRODUCTION}

Median sternotomy provides an excellent approach for cardiac surgery. It first was described by Milton in 1897 [Dalton 1992]. The incidence of sternal wound complications (SWC) ranges from $0.4 \%$ to $8 \%$, with a high morbidity

Received fuly 2, 2019; accepted October 3, 2019.

Correspondence: Abmed Abdelrabman Elassal, King Abdulaziz University Hospital, Cardiac Surgery Unit, Jeddah, Saudi Arabia, 21589; 00966566490807 (e-mail: samalassal1434@gmail.com). rate causing prolonged hospital stay and mortality rate of 14\% to 47\% [Thomas 2001; Bitkover 1998; Tavolacci 2003; Hirata 2003; Al-Ebrahim 2008]. Due to the recent increase of surgical options, it has become necessary to classify these wounds to assist the surgical decision-making process and facilitate the exchange of knowledge in scientific reports. The first classification was described by Pairolero and Arnold in 1984 [Pairolero 1984]. Based on our experience, we propose simple and more objective classification, regarding suitable treatment strategy rather than clinical and anatomical differentiation of the wound. Additionally, we consider the use of less invasive fasciocutaneous flap as an excellent reconstruction option to treat most sternal wound complications and even sternal bone loss [Anger 2012]. This saved patients from the unnecessary use of more invasive muscle or omental flaps, other major morbidities, or even mortality.

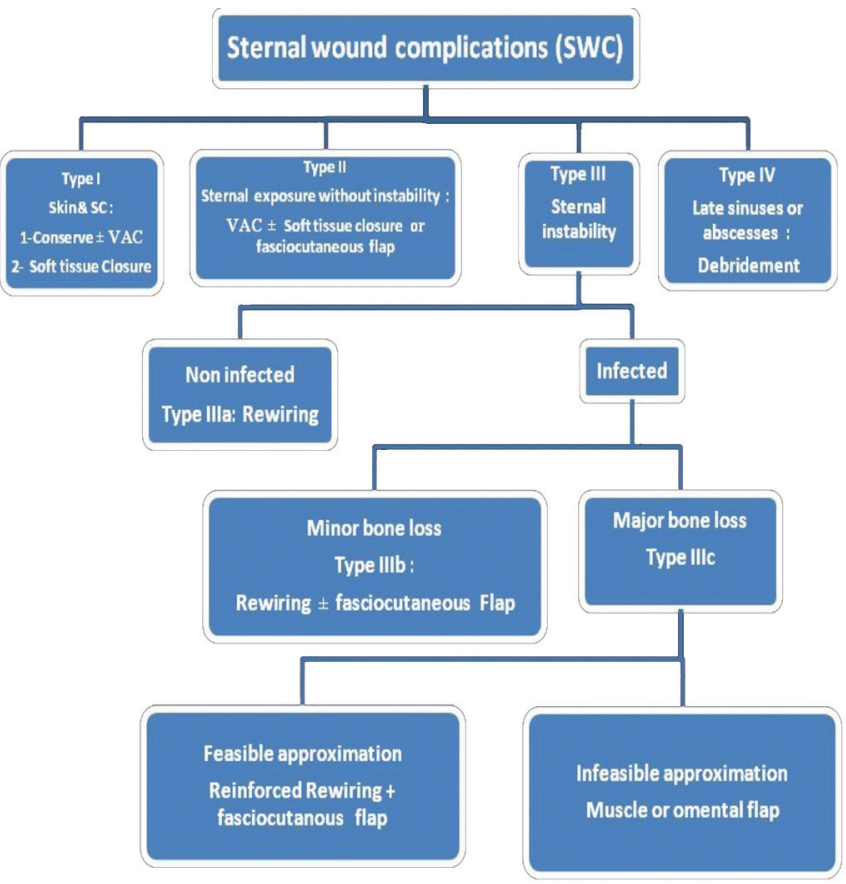

Authors' algorithm of post sternotomy wound complications and strategy of management. 
Table 1. Authors' Classification of post sternotomy wound complications and strategy of management

\author{
Type 1 \\ Description of the wound \\ Skin and subcutaneous tissue \\ Type II \\ Exposure of the sternum without \\ instability \\ Type III
}

A

B

C

Type IV

Chronicity: sinuses, abscesses

\begin{abstract}
Recommended management
Conservative management \pm VAC if failed Surgery:

debridement + direct soft tissue closure
\end{abstract}

VAC if failed Surgery: debridement + (direct soft tissue

closure or fasciocutaneous pectoral flap)

Non infected sternal instability

Infected sternal instability with minor bone loss

Infected sternal instability with major bone loss

\begin{abstract}
Surgery: rewiring
Surgery: debridement + rewiring + (direct soft tissue closure or fasciocutaneous pectoral flap)

Surgery: debridement + rewiring + fasciocutaneous pectoral flap for feasible wound approximation OR Muscle or omental flap for infeasible wound approximation
\end{abstract}

\section{MATERIALS AND METHODS}

From June 2006 to December 2018, 2,391 adult patients underwent full sternotomy during cardiac surgery in our hospital. One-hundred-sixteen patients with sternal wound complications retrospectively were reviewed; 86 of these cases conservatively were managed and the other 30 cases required surgical intervention. For infection prophylaxis, we used $1.5 \mathrm{~g}$ of first-generation cephalosporin (cefuroxime), which was administered during induction of anaesthesia, followed by $0.75 \mathrm{~g}$ every 8 hours. Vancomycin (500 $\mathrm{mg}$ ) intravenously was given to patients every 12 hours, if they were allergic to penicillin or cephalosporins. Assessment of SWC was based on clinical signs of sepsis, wound description, positive culture, CT findings of mediastinal collections, sternal instability, and surgical findings of sternal bone loss. We used Pairolero classification to look at the postoperative period that installs the infectious process in the surgical wound. Type I in the first week, Type II between 2 to 6 weeks, and Type III after 6 weeks to years (in general are fistulas and chronic osteomyelitis [Pairolero 1984]. We also applied classification proposed by Anger and colleagues based on anatomical site and depth of infection [Anger 2015]. These include Type I skin and subcutaneous tissue, Type II exposure of the sternum or ribs, Type III bone loss of sternum or ribs, and Type IV exposed mediastinum. We applied our own classification and algorithm; both are more objective to select the suitable therapy according to wound description (Table 1 and Figure 1). We mentioned only the specific main therapeutic modality for each class of SWC and neglected the nonspecific modalities, like preoperative preparation of the wound by conservative antimicrobial period with or without negative pressure wound therapy, to avoid dispersal and achieve focused and rapid access management.
Surgical decision making: Selection of surgical procedure mainly depends on detection of sternal exposure, sternal instability, extent of sternal bone loss, and feasible wound approximation, as shown in Table 1 and Figure 1. Our preferred reconstructive technique is the bilateral fasciocutaneous pectoral flap in sternal wound complications with exposed sternum, sternal bone loss, and even select cases of major bone loss. We consider major bone loss if greater than $50 \%$ of the sternum is affected with exposure of the mediastinum. We used it in 13 cases out of 30 surgical cases, reserving the use of more invasive other muscle or omental flaps for more complex cases with almost total sternal bone loss or infeasible wound approximation. Our selection of this technique depends on feasible wound approximation, preservation of residual sternal bone, and intact manubrium.

Technique: We carefully used dissecting scissors to undermine the bilateral pectoral fasciocutaneous flap, saving the pectoralis major muscle. The extent of dissection is defined, according to site of sternal bone loss and the possibility of approximation, in most cases lateral toward the midclavicular line and from suprasternal notch down to xiphoid. After good debridement, cleaning of the wound and rewiring, we closed the edges of the bilateral flaps together as one thick layer with transverse mattress tension sutures using polypropylene size 1 with simple interlacing 3/0 polypropylene stitches for skin. To enhance healing and adhesion of the flap to the sternum, we inserted a vacuum tube drain in front of the sternum and adhesive compression dressing to the wound. For more complex cases, we used the right rectus abdominis muscle flap in 2 cases and the bilateral pectoralis major flap in 1 case. Data obtained from medical records were compiled in an Excel spreadsheet and categorical variables presented as frequency and percentage. Continuous variables are presented as mean \pm standard deviation. 


\section{RESULTS}

The incidence of SWC in our center was $4.8 \%$. The perioperative patients' characteristics are shown in the second table (Table 2).

The mean duration to diagnose the SWC post primary operation was $37.04 \pm 86.35$ days. Computerized tomography (CT) was ordered for 10 patients for assessment of sternal stability and possible retrosternal collection, other diagnostic data is shown in Table 3 (Table 3 ).

Conservative management was adopted for 86 patients. The mean duration of conservative management was $11.19 \pm 9.8$ days. Surgical management was performed in 30 patients (25.86\%). Therapeutic measures are shown in Table 2.

Outcome after surgical management of SWC: Twentyeight $(93.3 \%)$ of our patients recovered and healed well after surgical intervention for SWC. Only 3 cases needed muscle flap, 1 pectoralis major and 2 rectus abdominis, however fasciocutaneous pectoral flap was performed for 13 cases with good outcomes. One exception was a recurrent case that was managed by the right rectus abdominis muscle flap (Figure 2). Two patients had recurrence - one conservatively was managed and the other was reoperated and healed well. The most common organisms identified with recurrent infections were N. coagulase (29.8\%), Klebsiella (12.5\%), pseudomonas $(10.5 \%)$, and MRSA (10.5\%). Other pathogens are shown in Table 3. Two cases were complicated because of inadvertent injury to the right ventricle, during dissection to release the sternum from the pericardium. We were obliged to conduct cardiopulmonary bypass through femoro-femoral cannulation, and both patients survived. We had 2 mortalities; neither was related to SWC. One was related to the cardiac procedure, and she was operated for CABG and had low cardiac output syndrome postoperatively. Intra-aortic balloon counterpulsation (IABP) was inserted, and the chest left open for 3 days. She developed SWC, and MRSA was isolated from wound culture. Rewiring and pectoral muscle flap were done. Unfortunately, this patient died from cardiogenic shock 27 days postoperatively. The other case died from septic shock, due to an aggressive chest infection.

\section{DISCUSSION}

Deep sternal wound complications represent a significant problem in open heart surgery and pose a challenge to surgeons. The dehiscence and infection after median sternotomy increases the patient's morbidity and mortality [Thomas 2001; Bitkover 1998; Tavolacci 2003; Hirata 2003; Al-Ebrahim 2008]. Reported common risk factors include old age, use of bilateral mammary artery, prolonged surgery, low cardiac output, reoperation for bleeding, prolonged mechanical ventilation, diabetes mellitus, obesity, and chronic obstructive pulmonary disease [Dalton 1992; Thomas 2001; Bitkover 1998; Balachandran 2016]. In our study, female gender, DM, obesity, COPD, and long ICU stay were frequent common risk factors. Uncommon risk factors identified included tracheostomy, delayed sternal closure, refractory cough, and excessive blood transfusion. N. coagulase, Klebsiella, pseudomonas, and MRSA represented the most common organisms

Table 2. Perioperative patients' characteristics

\begin{tabular}{|c|c|}
\hline Variable & Value \\
\hline Age, mean $\pm S D$ & $62 \pm 10.36$ \\
\hline $\operatorname{Sex} F / M$ & $53 / 63$ \\
\hline Mean $\mathrm{BMI} \pm \mathrm{SD}$ & $29.12 \pm 4.79$ \\
\hline DM & $105(90.5 \%)$ \\
\hline Hypertension & $86(74.13 \%)$ \\
\hline Dyslipidemia & $84(72.41 \%)$ \\
\hline Smoking & $38(32.75 \%)$ \\
\hline Creatinine $>115 \mu \mathrm{mol} / \mathrm{L}$ & $39(33.62 \%)$ \\
\hline Immunocompromised & 6 \\
\hline On corticosteroids & 8 \\
\hline COPD & 11 \\
\hline Effusions & 6 \\
\hline \multicolumn{2}{|l|}{ Primary surgical procedure } \\
\hline CABG & $113(97.4 \%)$ \\
\hline $\mathrm{CABG} \pm \mathrm{AVR}$ & 1 \\
\hline Other procedures & 2 \\
\hline \multicolumn{2}{|l|}{ Pedicled ITA use } \\
\hline None & 2 \\
\hline Single & 107 \\
\hline Bilateral & 7 \\
\hline Mean aortic cross clamp time (min.) \pm SD & $75.9 \pm 30.6$ \\
\hline Mean bypass time (min.) $\pm \mathrm{SD}$ & $113.55 \pm 36.14$ \\
\hline Unit of transfused packed RBCs mean \pm SD & $4.7 \pm 2.7$ \\
\hline Duration of MV (days) & $3.5 \pm 3.5$ \\
\hline Tracheostomy & 2 \\
\hline Delayed sternal closure (DSC) & 3 \\
\hline LCOS (low cardiac output syndrome) & 6 \\
\hline IABP & 25 \\
\hline Refractory idiopathic cough postop & 4 \\
\hline ICU stay & $7.6 \pm 6.46$ \\
\hline Hospital stay & $32.9 \pm 30.01$ \\
\hline \multicolumn{2}{|l|}{ Management of SWC } \\
\hline Conservative management & 86 \\
\hline VAC & 36 \\
\hline Rewiring & 21 \\
\hline Reconstruction total & 16 \\
\hline Fasciocutaneous pectoral flap & 13 \\
\hline Pectoral muscle flap & 1 \\
\hline Rectus abdominis muscle flap & 2 \\
\hline
\end{tabular}


and identified in $63.4 \%$ of positive cultures. We could not find a correlation between the type of causative organisms and a particular risk factor. Sternal wound complications are among the most preventable ones in cardiac surgery when the necessary precautions are taken and high-risk patients are identified and primary reinforced sternal closure is implemented [Al-Ebrahim 1996; Robicsek 2000; Al Ebrahim 2003; Baskett 1999]. We diagnosed SWC in 30 patients (25.8\%), during the same admission. Another 86 cases were presented after hospital discharge and necessitated careful management

Table 3. Diagnostic data

\begin{tabular}{|c|c|}
\hline Diagnosis in the same admission for primary operation & $30(25.87 \%)$ \\
\hline Diagnosis after discharge post primary operation & $86(74.13 \%)$ \\
\hline Mean duration (days) $\pm S \mathrm{~S}$ & $37.04 \pm 86.35$ \\
\hline \multicolumn{2}{|l|}{ Anger classification } \\
\hline 1 & 68 \\
\hline II & 31 \\
\hline III & 8 \\
\hline IV & 9 \\
\hline \multicolumn{2}{|l|}{ Pairolero classification } \\
\hline 1 & 33 \\
\hline II & 76 \\
\hline III & 7 \\
\hline \multicolumn{2}{|l|}{ Our classification } \\
\hline 1 & 64 \\
\hline II & 30 \\
\hline III (A) & 1 \\
\hline III (B) & 11 \\
\hline III (C) & 10 \\
\hline \multicolumn{2}{|l|}{ CT for diagnosis of infection (ordered for 10 patients) } \\
\hline Within normal & 1 \\
\hline Sternal instability with no evidence of mediastinitis & 1 \\
\hline Sternal instability with evidence of mediastinitis & 8 \\
\hline Intraoperative signs of sternal osteomyelitis & 21 \\
\hline \multicolumn{2}{|l|}{$\begin{array}{l}\text { Pathogens identified by culture from wound discharge or } \\
\text { mediastinal fluids }\end{array}$} \\
\hline Heamophilus & 4 \\
\hline N. Coagulase Staph & 31 \\
\hline Pseudomonas & 11 \\
\hline Acinobacter & 7 \\
\hline Klebsiella & 13 \\
\hline MRSA & 11 \\
\hline E. coli & 5 \\
\hline Pneumococci & 10 \\
\hline Staph. Aureus & 8 \\
\hline Staph. Epidermides & 4 \\
\hline
\end{tabular}

and regular follow up particularly for patients with risk factors. Due to the recent increase of surgical options, it became necessary to classify these wounds to assist the surgeon decision-making process regarding technique, and to facilitate the exchange of knowledge in scientific reports. Several classifications were reported, describing post sternotomy mediastinitis, according to postoperative period, extent of infection, and strategy of management [Pairolero 1984; Oakley 1996; Greig 2007; Anger 2007]. The first classification was described by Pairolero and Arnold in 1984 [Pairolero 1984], based on the postoperative time of establishment of the infection. Subsequently, Oakley in 1996 [Oakley 1996] used the same criteria, but added risk factors of the establishment and attempts of treatment of the initial infection. Jones et al [Greig 2007] in 1997, suggested for the first time a classification based on the affected anatomical site, but still using parameter as the presence of infection. Greig et al [Greig 2007] proposed a classification based on the affected anatomical site. Anger and colleagues were the first to specify the vertical extent of the wound because it recognized the increased difficulty to reconstruct the lower portion when it extends below the insertion of the lower border of the pectoralis major muscle [Anger 2007]. We consider terminology like mediastinitis, superficial, and deep-wound infections to be subjective and nonspecific descriptions. They have dispersed relation to the targeted management, which requires more objective, defined, and conclusive tissue wound description. We applied our own classification and algorithm, which are more objective, to select the proper management according to simple and focused tissue wound description (see Table 1 and Figure 1). That helps rapid access of strategy of management, which avoids late and conflicting decision-making. Negative pressure wound therapy decreases wound oedema, accelerates wound healing and granulation tissue formation, increases sternal blood flow, and has been shown to improve early- and long-term survival in patients with deep sternal wound infection [Debreneci 2008]. We applied VAC in 36 patients (31\%) as a sole therapy or as a preliminary step before surgery. For the past 40 years, clinical trials have recommended the use of vascularized tissue to fill in the defect in the sternal region and to reduce the possibility of infection and that decreases morbidity when employing muscle or omentum flaps [Anger 2012]. Surgeons adopt a policy of liberal use of muscle flaps based on the depth of infection and magnitude of sternal bone loss. However, pain, limitation of physical activities, large detachments, problems in donor areas, and risk of spread of infection were not uncommon complications [Anger 2012; Davison 2007; Reade 2003; vanWingerden 2011]. So the need for less invasive and less complicated technique is a better choice. The cornerstone of our management is the ability to approximate the surgical wound, even in cases of major bone loss, so long as there are residual sternal edges. We think the possibility to approximate the wound without tension rather than the degree of bone loss determines the need for muscle flap reconstruction. In these cases, we advocate use of the less invasive pectoral fasciocutaneous flap and avoiding unnecessary use of the more invasive muscle or omental flaps. More than $40 \%$ of our cases were managed 
using fasciocutaneous flaps with good results. There was only one failure that required rectus flap.

In conclusion, sternal wound complications are devastating events and there is no consensus or clear guidelines to clarify classification or determine conclusive management. Objective wound classification is helpful to organize the range of sternal wound complications and enables us to adopt the proper treatment strategy. Use of fasciocutaneous pectoral flap is a favorable and less invasive modality for most reconstructive procedures, and it avoids unnecessary use of the more invasive muscle or omental flaps.

\section{ACKNOWLEDGEMENT}

This project was funded by the Deanship of Scientific Research (DSR), King Abdulaziz University, under grant No. (DF-821-140-1441). The authors, therefore, gratefully acknowledge the DSR technical and financial support.

\section{REFERENCES}

Al-Ebrahim K. 2003. Reinforced sternal closure; the bilateral straight longitudinal wire technique. Asian Cardiovasc Thorac Ann. 11: 90-91.

Al-Ebrahim K. 2008. Simplified modified reinforced sternal closure; European Journal of Cardio-Thoracic Surgery. 34(5): 1127-1128.

Al-Ebrahim K, Shafei, H. 1996. A new technique for reinforced sternal closure. Asian Cardiovasc Thorac Ann 4: 117-8.

Anger J, Dantas D, Arnoni R, Farsky P. 2015. A new classification of post-sternotomy dehiscence. Rev Bras Cir Cardiovasc. 30(1): 114-118.

Anger J, Farsky PS, Almeida AF, Arnoni RT, Dantas DC. 2012. Use of the pectoralis major fasciocutaneous flap in the treatment of post sternotomy dehiscence: a new approach. Einstein. 10(4):449-454.

Balachandran S, Lee A, Denehy L, Lin KY, Royse A, Royse C, and ElAnsary D. 2016. Risk Factors for Sternal Complications After Cardiac Operations: A Systematic Review. Ann Thorac Surg 102:2109-17.

Baskett R, Mac Dougall C, Ross D. 1999. Is mediastinitis a preventable complication? A 10-year review. Ann Thorac Surg. 67:462-5.

Bitkover C, Gardlund B. 1998. Mediastinitis after cardiovascular operations: a case-control study of risk factors. Ann Thorac Surg. 65:36-40.

Dalton ML, Connally SR, Sealy WC. 1992. Julian's reintroduction of Milton's operation. Ann Thorac Surg. 53:532-3.

Davison SP, Clemens MW, Armstrong D, Newton ED, Swartz W. 2007. Sternotomy wounds: rectus flap versus modified pectoral reconstruction. Plast Reconstr Surg. 120:929-34.

Debreneci T, Szerafin T, Galajda Z, Miskolczi S, Peterfly A. 2008. Results of vacuum-assisted wound closure system in the treatment of sternotomy wound infections following cardiac surgery. Magyar Sebeszet. 61:10.1556.

Greig AV, Geh JL, Khanduja V, Shibu M. 2007. Choice of flap for the management of deep sternal wound infection--an anatomical classification. J Plast Reconstr Aesthet Surg. 60(4):372-378.

Hirata N, Hatsuoka S, Amemiya A, Takayoshi U, Kosakai Y. 2003. New strategy for treatment of MRSA mediastinitis: one-stage procedure for omental transposition and closed irrigation. Ann Thorac Surg. 2003; 76:2104-6.

Oakley E, Wright J. 1996. Postoperative mediastinitis: classification and management. Ann Thorac Surg. 61:1030-6.

Pairolero PC, Arnold PG. 1984. Management of recalcitrant median sternotomy wounds. J ThoracCardiovasc Surg. 88(3):357-364.

Reade CC, Meadows WM, Jr, Bower CE, et al. 2003. Laparoscopic omental harvest for flap coverage in complex mediastinitis. Am Surg. 69:1072-6.

Robicsek F, Fokin A, Cook J, Bhatia D. 2000. Sternal instability after midline sternotomy. ThoracCardiovasc Surg. 48:1-8.

Tavolacci M, Merle V, Josset V, Bouchart F, Litzler P, Tabley A. 2003. Mediastinitis after coronary artery bypass graft surgery: influence of the mammary grafting for diabetic patients. J Hosp Infect. 55:21-5.

Thomas J, Kouchoukos N. 2001. A rational approach to wound difficulties after sternotomy: the problem. Ann Thorac Surg. 72: 1411-8.

vanWingerden JJ, Lapid O, Boonstra PW, de Mol BA. 2011. Muscle flaps or omental flap in the management of deep sternal wound infection. Interact Cardiovasc Thorac Surg. 13:179-87. 